\title{
CLASSIFYING IMMERSED CURVES
}

\author{
J. SCOTT CARTER
}

(Communicated by Frederick R. Cohen)

\begin{abstract}
Let a collection $\gamma$ of generically immersed curves be given in an oriented surface $G$. To each component circle, associate a Gauss word by traveling once around the circle and recording the crossing points with signs. The set of these words forms a Gauss paragraph. If $\gamma_{1}$ and $\gamma_{2}$ fill the surface $G$ in the sense that the complementary regions are disks, then there is a homeomorphism of $G$ taking one to the other if and only if $\gamma_{1}$ and $\gamma_{2}$ have isomorphic Gauss paragraphs. This notion of isomorphism is defined here; it ignores the choices made in defining the Gauss words.
\end{abstract}

\section{INTRODUCTION}

(1.1) Motivation. Immersed curves are interesting. Despite their apparent simplicity, they admit a wonderful variety of invariants. One of these invariants, the Gauss word (or paragraph if the domain has more than one component), classifies curves that fill a surface. The complement in a surface of a filling collection of curves is a collection of disks. This classification theorem is the main result of the paper. It will be used extensively in the companion paper [4].

Reading [2] or [3], either before this paper or in conjunction with it, may be helpful.

(1.2) History. Many authors have found immersed curves a fruitful subject to study. Here is a condensed history. Gauss [10] considered finite sequences of letters that have the property that every letter appears exactly twice. The projection of a knot possesses such a sequence. He mused about when such a sequence would give the projection of a knot. This problem was solved by Treybig [15], Marx [12], Lovasz and Marx [11], Read and Rosenstiehl [14], and Dowker and Thistlethwaite [7]. In these works necessary and sufficient criteria were established that determine the "planarity" of a Gauss word. Whitney [16] showed how to break the crossings of an immersed planar curve to compute its winding number. Chillingsworth [5], [6] generalized Whitney's results to immersed curves in arbitrary surfaces. He showed how winding numbers could be associated to elements of the fundamental group. Extensions of immersed

Received by the editors October 2, 1989.

1980 Mathematics Subject Classification (1985 Revision). Primary 57M20. 
curves in the sphere to maps of surfaces into the sphere were studied by several authors including Bailey [1], Blank as reported in [13] and Francis [8].

C. F. Bodigheimer originally told me of Gauss's manuscript in the summer of 1987. I have since become enamored with the subject of immersed curves.

(1.3) The classification theorem. The result proven here is the following:

Theorem. Stable geotopy classes of immersed curves correspond to isomorphism classes of Gauss paragraphs.

The terminology is established in $\S \S(2.1),(2.2)$, and (2.3). Section (2.1) shows how to associate a Gauss paragraph to a generically immersed collection of curves. Section (2.2) defines a Gauss paragraph in abstract and defines an equivalence relation on the set of these paragraphs. In $\S(2.3)$, the notion of stable geotopy is defined.

In $\S(3.1)$, a cell complex that is canonically associated to a Gauss paragraph is constructed. The proof of the theorem then appears in $\S(3.2)$.

Section 4 summarizes the companion paper.

(1.4) Conventions. The following notations are consistently used: An immersed curve $\gamma: \bigsqcup_{j=1}^{k} S_{j}^{1} \rightarrow G$ of $k$-components has as its image the oriented closed surface $G$. Manifolds of dimension 2 are called surfaces. The curve $\gamma$ is generically immersed; so each double point has a neighborhood such that the image of $\gamma$ in this neighborhood looks like the intersection of the coordinate axes in the 2-disk. Thus the word immersed always means generically immersed. Lower case Roman letters, (with subscript) correspond to the crossing points of the given curves.

\section{DEFINITIONS}

(2.1) Gauss paragraphs associated to immersed curves. Let a curve in a surface be given. Say, $\gamma: \coprod_{j=1}^{k} S_{j}^{1} \rightarrow G$ is a generic immersion into the oriented surface $G$. Label the double points (in the surface $G$ ) of the curve $\gamma$ by elements in the set $A=\left\{a_{1}, \ldots, a_{n}\right\}$. The singular set of $\gamma=\left\{x \in \coprod_{j=1}^{k} S_{j}^{1}:\left|\gamma^{-1} \gamma(x)\right|=2\right\}$ can be put into one-to-one correspondence with the set $A^{ \pm 1}=\left\{a_{1}^{ \pm 1}, \ldots, a_{n}^{ \pm 1}\right\}$. Then a Gauss word (or Gauss paragraph if $k>1$ ) is associated to the curve by traversing each component of $\gamma$ exactly once and recording the double points in order by juxtaposing the corresponding letter in $A$ (with an exponent) to the right end of the word associated to the given component. The exponent is +1 if the other sheet crosses from left to right at the given double point; otherwise the exponent is -1 . Left and right are determined by the orientation of the image surface $G$.

As defined here, each immersed curve has several Gauss paragraphs associated to it since five choices are made in the definition: (1) base point of each curve, (2) orientation of each curve, (3) labeling the double points, (4) the sign convention for crossings, and (5) permuting the order of the component circles. 
(2.2) Abstract Gauss paragraphs. A Gauss paragraph is defined in the abstract as a pair $(P, f)$ where $P$ is a partition of the set $\{1,2, \ldots, 2 n\}$ and the mapping $f:\{1,2, \ldots, 2 n\} \rightarrow\left\{a_{1}^{ \pm 1}, \ldots, a_{n}^{ \pm 1}\right\}$ is a one-to-one correspondence. The words of a Gauss paragraph are the images $f(p)$, where $p$ is a set in the partition $P$.

Two Gauss paragraphs are said to be isomorphic if one can be obtained from the other by a combination of (1) cyclically permuting the sequence of any word; (2) reversing the sequence of any words; (3) permuting the alphabet set $A$; (4) changing all the exponents from + to - and vice versa; (5) permuting the order in which the words appear. The allowable moves of abstract Gauss paragraphs correspond, respectively, to the choices made in associating a Gauss paragraph to an immersed curve.

Dan Frohardt [9] pointed out that the isomorphism classes of Gauss paragraphs that contain only one word can be put in one-to-one correspondence with double cosets of the symmetry group $\sum_{2 n}$ where one subgroup is an embedded $\sum_{n} \times \mathbf{Z} / 2$ and the other is an embedding of a dihedral group of order $4 n$. The dihedral group is acting classically on the (cyclic) Gauss word. He has found a formula [9] to compute the number of isomorphism classes of Gauss words. Of course, the set of isomorphism classes of arbitrary Gauss paragraphs can similarly be put into correspondence with a set of double cosets; the counting becomes more difficult in this case.

(2.3) Stable geotopy. Two immersed curves,

$$
\gamma_{1}: \coprod_{j=1}^{k} S_{j}^{1} \rightarrow G_{1} \text { and } \gamma_{2}: \coprod_{j=1}^{k} S_{j}^{1} \rightarrow G_{2},
$$

each with $k$ components are geotopic if and only if there is a homeomorphism, $h: G_{1} \rightarrow G_{2}$, such that $h \gamma_{1}=\gamma_{2}$. The curves are said to be stably geotopic if and only if there is a collection of hollow 1-handles that can be added to either surface, or both, such that the curves are geotopic on the resulting surfaces. The attaching regions for these handles are disjoint from the image of the immersed curve. Equivalently, the curves are stably geotopic if there is a collection of 2-handles to be taken from the surfaces such that the curves are geotopic on the results of these surgeries.

From this equivalent definition and Theorem (1.3), Gauss paragraphs classify filling immersed curves up to a homeomorphism of the surface.

\section{THE PROOF OF THE THEOREM}

The proof will follow the construction of a cell complex associated to an abstract Gauss paragraph. This complex will turn out to be the minimal genus surface in which a curve with the given Gauss paragraph can be immersed. The surface in which a given curve is immersed will be surgered to be homeomorphic to the surface associated to the Gauss paragraph. Then a homeomorphism will be constructed that takes the given curve to a standard. 

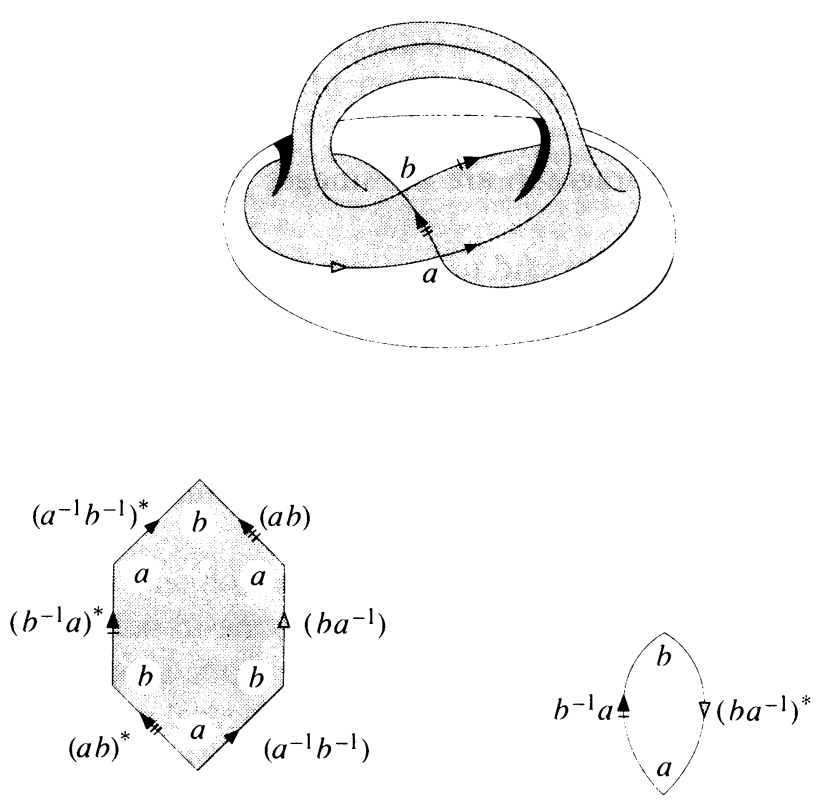

Figure A

(3.1) The cell complex associated to a Gauss paragraph. Let an abstract Gauss paragraph be defined on the finite alphabet set $A=\{a, b, \ldots\}$. The paragraph is a partitioned permutation of the set $\left\{a^{ \pm 1}, b^{ \pm 1}, \ldots\right\}$. Here is the construction of a 2-complex that is associated to the Gauss paragraph. The vertices are the elements of the set $A$. The oriented edges are the two-letter syllables found in the cyclic Gauss words of the paragraph. If a given word in the paragraph has exactly one letter, say $a$, then there is an edge $a a$.

Here is an example in which the paragraph has one word: $w=a b a^{-1} b^{-1}$. The vertices of the complex are the letters $a$ and $b$. The edges are the pairs $a b, b a^{-1}, a^{-1} b^{-1}$, and $b^{-1} a$. In Figure A, the complex associated to the word $w$ is illustrated.

The faces of the complex will be described by specifying the edges in the complex to which they are attached. A segment of the boundary of a face is attached to an edge in either an orientation-preserving or an orientationreversing fashion. In fact, each edge will appear in the union of the boundaries of the faces exactly twice, once forward and once backward.

Suppose that $E=x^{ \pm 1} y^{ \pm 1}$ is a two-letter syllable of some Gauss word. Let $E^{*}=\left(x^{ \pm 1} y^{ \pm 1}\right)^{*}$ denote the edge with opposite orientation. Consider the set $\mathscr{E}$ that is the union of all edges, $E$, and all opposite edges, $E^{*}$. In $\mathscr{E}$, each vertex is mentioned exactly four times. A given element $E \in \mathscr{E}$ approaches a vertex from one of four compass directions: the direction is determined by whether or not $E$ is starred and by the exponent on $x$ or $y$. Specifically, $x^{ \pm 1} y$ approaches $y$ from the south; $x^{ \pm 1} y^{-1}$ approaches $y$ from the west; $\left(x y^{ \pm 1}\right)^{*}$ 


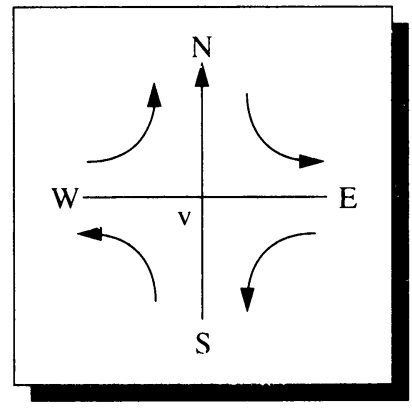

Always turn left.

FIGURE B

approaches $x$ from the north; and $\left(x^{-1} y^{ \pm 1}\right)^{*}$ approaches $x$ from the east. A terminal vertex of an element $E \in \mathscr{E}$ is the vertex being approached.

Suppose a (possibly empty) set of faces has been constructed. Pick an element $E \in \mathscr{E}$ that is not on the boundary of any of the previous faces. The face that contains $E$ has as its boundary the (cyclic) sequence of edges obtained from $E$ by successively turning left at terminal vertices. Left turns are determined by the table below; see also Figure $B$.

\begin{tabular}{|ll|}
\hline the edge $S v$ & is followed by $\left(W v^{-1}\right)^{*} ;$ \\
the edge $W v^{-1}$ & is followed by $v N ;$ \\
the edge $\left(v^{-1} E\right)^{*}$ & is followed by $v^{-1} E ;$ \\
the edge $(v N)^{*}$ & is followed by $(S v)^{*}$
\end{tabular}

The surface that is associated to a Gauss paragraph is the cell complex that was just constructed: vertices $\leftrightarrow\{a, b, \ldots\}$, edges $\leftrightarrow$ \{two letter syllables $\}$, and the boundaries of faces are found by successive left turns. The boundary of any face is called a cycle in the Gauss paragraph. It is not difficult to see that this is a surface: each vertex is at the end of four edges, and each edge is on the boundary of two faces.

(3.2) The Proof of (1.3). If two immersed curves are stably geotopic, then they have isomorphic Gauss paragraphs. Geotopies cannot change the intersection information of the curve.

Let an immersed curve $\gamma$ be given in a surface $G$. Construct the surface associated to the Gauss paragraph of $\gamma$. Consider the map from the set of cycles in the Gauss paragraph to the surface $G$ that is given by the immersion $\gamma$. Approximate the image of a cycle by an embedded circle disjoint from the image of the immersion $\gamma$. If this approximating circle represents some homotopy, then the surface $G$ can be surgered along the circle. This process is performed for each cycle in the Gauss paragraph until each region determined by the cycles is simply connected. The resulting surface is homeomorphic to the surface associated to the Gauss paragraph.

The faces of the surface associated to the Gauss paragraph are a collection 
of polygons. In the surgered surface containing $\gamma$, the complement of $\gamma$ is also a set of polygons; the boundary of each is a cycle in the Gauss paragraph. A homeomorphism is constructed between these surfaces by taking corresponding polygons to each other; the correspondence is determined by the bounding cycle. Thus any two immersed curves with isomorphic Gauss paragraphs can both be obtained from the surface associated to their common Gauss paragraph by attaching 1-handles. This completes the proof.

\section{Summary of the companion PAPER}

In [4], Gauss words are associated to properly immei sed arcs. Necessary and sufficient criteria are given in terms of these bracketed words that allow one to conclude when a collection of properly immersed arcs form the singular set of a proper immersion. The types of triple points that can occur in a threedimensional manifold are classified. Genera are associated to immersed curves and pairings of their double points. These genera represent optimal immersions that achieve the given pairings. They measure the complexity of the given curves.

\section{ACKNOWLEDGMENTS}

This work was completed while I visited Wayne State University with the assistance of a Summer Research Award. The foundations were laid during my visit to the University of Texas at Austin in 1987-1988. Both institutions were hospitable. I had several conversations with Dan Frohardt and Bob Bruner which were helpful. Thanks go to those folks who have encouraged me to continue my work; also I thank David Byrne and Robert Pirsig.

\section{BIBLIOGRAPHY}

1. K. D. Bailey, Extending closed plane curves to immersions of the disk with $n$ handles, Trans. Amer. Math. Soc. 206 (1975), 1-24.

2. J. Scott Carter, Extending immersed circles in the sphere to immersed disks in the ball, preprint.

3. __ Examples of properly immersed disks in the 3-ball, preprint.

4. __ Extending immersions of curves to properly immersed surfaces, Topology Appl. (to appear).

5. D.R.J. Chillingsworth, Winding numbers on surfaces I, Math. Ann. 196 (1972), 218-249.

6. __ Winding numbers on surfaces II, Math. Ann. 199 (1972), 131-153.

7. C. H. Dowker, and Morwen B. Thistlethwaite, Classification of knot projections, Topology Appl. 16 (1983), 19-31.

8. George Francis, Extensions to the disk of properly nested plan immersions, Michigan Math. J. 17 (1970), 377-383.

9. Daniel Frohardt, On equivalence classes of Gauss words, preprint.

10. C. F. Gauss, Werke VIII, pp. 271-286.

11. L. Lovasz and M. L. Marx, A forbidden substructure characterization of Gauss codes, Acta Sci. Math. 38 (1976), 115-119.

12. M. L. Marx, The Gauss realizability problem, Proc. Amer. Math. Soc. 22 (1969), 610-613. 
13. V. Poenaru, Extensions des immersions en codimension 1 (d'aprés Blank), Seminar Bourbaki, 1967/68), Expose 342, Benjamin, New York (1969).

14. R. C. Read and P. Rosenstiehl, On the Gauss crossing problem, Colloq. Math. Soc. János Bolyai, North-Holland, Amsterdam and New York, 1976, pp. 843-876.

15. L. B. Treybig, A characterization of the double point structure of the project of a polygonal knot in regular position, Trans. Amer. Math. Soc. 130 (1968), 223-247.

16. H. Whitney, On regular closed curves in the plane, Compositio Math. 4 (1937), 276-284.

Department of Mathematics, University of South Alabama, Mobile, Alabama 36688 